\title{
Discrete-Impulse Energy Supply in Milk and Dairy Products Processing
}

\author{
Paweł Droździel ${ }^{1}$, Tetiana Vitenko ${ }^{2}$, Viktor Voroshchuk ${ }^{3}$, Sergiy Narizhnyy ${ }^{4}$, Olha Snizhko ${ }^{5}$
}

1 Paweł Droździel; Lublin University of Technology, Faculty of Mechanical Engineering, Nadbystrzycka Street 36, 20-618 Lublin, Poland

2 Tetiana Vitenko; Department of Food Technology Equipment, Faculty of Engineering of Machines, Structures and Technologies, Ternopil Ivan Puluj National Technical University, Ukraine

3 Viktor Voroshchuk; Department of Food Technology Equipment, Faculty of Engineering of Machines, Structures and Technologies, Ternopil Ivan Puluj National Technical University, Ukraine

4 Sergiy Narizhnyy; Department of Food Technology and Technology Processing of Animal Products, Faculty of Biotechnological, Bila Tserkva National Agrarian University, Ukraine

5 Olha Snizhko; Department of Technologies of Meat, Fish and Marine Products, Faculty of Food Technologies and Quality Management of Products of Agricultural Produkts, National University of Life and Environmental Scienses of Ukraine, Ukraine

* Correspondence: p.drozdziel@pollub.pl

\begin{abstract}
The basis of the discrete-impulse energy supply (DIES) concept is the efficient use of supplied energy. The references describe in detail the general principles of DIES, examine the energy and thermodynamic aspects and the main mechanisms of intensification that can be initiated on the basis of this principle. DIES mechanisms conveniently can be divided into hard and soft ones. The former should be used to stimulate hydromechanical processes, and the latter to accelerate the processes of phase heat and mass transfer, or for the purpose of intensive mixing of multicomponent media. The authors have studied the possibility of using DIES to intensify the hydromechanical processes, in particular emulsification of milk fat (homogenization of milk, preparation of spreads), processing of cream cheese masses. Objects of research were whole non-homogenized milk, fat emulsions, cream cheese mass. In order to evaluate the efficiency of milk homogenization the homogenization coefficient change was studied, which was determined by centrifugation method as the most affordable and accurate one. Emulsions were evaluated according to the degree of destabilization, resistance and dispersion of the fat phase. The rheological characteristics of cheese masses were evaluated by the effective viscosity change.
\end{abstract}

Keywords: discrete-impulse energy, hydromechanic, process, milk products

\section{Introduction}

The development of energy-saving technologies that meet the modern production requirements and provide optimum conditions for the processes is usually based on the development of new conceptions. The improvement of the most widespread and energyintensive processes has remained an urgent issue for the food industry and dairy processing in particular. Such processes as mixing, homogenizing, emulsifying can be used as an example of energy-intensive technologies. As a rule, the intensification of such processes in multicomponent environments takes place due to the supply of external energy, which is introduced into the apparatus by mechanical stirring, the introduction of additional streams of liquid or gas, the initiation of vibrations, the use of centrifugal forces, acoustic or pulsed influences, and powerful electrical discharge. All these methods contribute to the forced relative motion of phases, provide deformation and grinding of dispersed particles, increase particles period of stay in the volume of the apparatus and equal distribution of the dispersed phase into a continuous medium. At the same time, these methods are limited in their effectiveness. Since effective grinding is performed mainly within the energy supply area, the continuous medium and dispersed inclusions are 
mixed as one unit in the volume of the apparatus, and a significant part of the energy is unproductively consumed to overcome viscous forces and frictional forces. In order to use energy effectively it is necessary to provide a certain level of power for a short period of time (to convert energy into a form of short, powerful pulses). Such energy supply in the scientific literature was called a discrete-impulse energy supply (DIES) [1-3].

The general principles of DIES, the energy and thermodynamic aspects and the main mechanisms of intensification that can be initiated on the basis of this principle have been described in the papers in detail [2], [4 - 7]. In the opinion of the authors [8], it is necessary to create a large number of vapor or gas-vapor bubbles in order to implement the conditions for the discrete energy distribution in the working fluid volume of the fluid medium. They can be considered as microtransformers, which convert the potential energy accumulated in the system into kinetic one, which is discretely distributed in space and time. DIES mechanisms conditionally can be divided into hard and soft ones. The former should be used to stimulate hydromechanical processes, and the latter to accelerate the processes of phase heat-mass transfer, or for the purpose of intensive mixing of multicomponent media, when the level of intensification of the process can be smoothly changed within certain limits. In practice, such conditions are obtained by ultrasonic influence on the liquid [9] or by the creation of special hydrodynamic conditions in the flow [10] in the propellant or rotor apparatus.

The problem of the intensification of hydromechanical processes in milk and dairy processing is extremely important today. The ways of solving this problem can be different. Traditional methods of intensification are based on the concept of local isotropic turbulence, the basis of which is in [11] and developed by the authors [12, 13]. In accordance with the statements of this theory, an important role in stimulating hydromechanical particles grinding is played by pulsations of velocity fluctuation of the turbulent flow $v^{\prime}$ and p'. The size of the maximum droplets formed during the grinding in the emulsification processes according to the theory of local isotropic turbulence is determined from the equation $d_{\max }=\frac{\sigma}{\rho_{c} v^{\prime 2}}$ and depends on the coefficient of interphase tension, the values of the turbulent pulsations and the density of the medium. From an energy point of view, the level of influence intensification is characterized by the size of the local power dissipation per fluid mass unit. Therefore, to increase the level of intensification, it is necessary to obtain maximum values of pulsation velocity. Moreover, the maximum effect is possible in the areas with the highest power dissipation. For this purpose they usually increase the speed of the flow in the pipelines, the speed of the mixer rotation, the roughness of the walls, use buffles, diaphragms, turbulizing gratings, etc. [14-16]. It should be noted that, along with an increase in the value of the supplied energy, the efficiency of its use decreases as its main part is unproductively dissipated in the volume of the continuous phase and on the walls of the apparatus. The authors [17] explained in detail that controlling the processes of hydromechanical emulsification or homogenization based solely on the theory of local isotropic turbulence is not correct since the physical mechanism of the dispersion process is not related to the direct action of turbulent pulsations. Other researchers [18] describe a mathematical model for deformation and droplets destruction in liquid and gaseous media based on shear stresses and accelerated flows.

The principle of shock impact is based on the explosive effect of the cumulative current (accumulation of energy and its realization in a short period of time on a small space). The high value of specific power within the area under treatment is reached by reducing the spatial temporal localization of energy, which is possible in the volume of saturated vapor-gas bubbles. In the process of bubbles growth or compression in fluid some nonstationary micro flows are formed around them which create a dynamic effect on the disperse particles. The results presented in $[19,20]$ show that the velocity gradients of radial microcurrents around bubbles reach 107s-1, acceleration exceeds 106g, and the value of the pressure pulse at the boundary with the bubble reaches $1000 \mathrm{MPa}$, which can lead to the destruction of solid dispersions. Among the mechanisms of DIES, which we will further rely on, the cavitational mechanisms, which provide the formation of a significant number of vapor-gas bubbles and the effect of shear stresses should be mentioned. These 
mechanisms can be introduced in static/dynamic cavitation apparatus, rotary pulsating apparatus, etc [21 - 23]. Their use in milk processing technologies is quite promising and important from the point of view of the introduction of innovative technologies into production.

The authors have studied the possibility of using cavitation and rotary-pulsating apparatus aimed at intensification of hydromechanical processes, in particular emulsification of milk fat (milk homogenization, spreads preparation), processing of cream cheese masses.

\section{Materials and Methods}

\subsection{The object and methods of estimation of the homogenized milk quality.}

The objects of research were whole non-homogenized milk, fat emulsions, cream cheese mass.

The whole non-homogenized milk with fat content of $4 \%$, with titrated acidity of 16 $20 \mathrm{~T}$, homogeneous consistency, without precipitates and flakes, off-flavor, white color was used as an object of research of cavitation effects on milk fat. To evaluate the efficiency of homogenization Gerber method was used. It is based on extraction of fat from milk and dairy products under the influence of concentrated sulfuric acid and isoamyl alcohol, followed by centrifugation and measuring of its volume by graduated part of the pioscope. Before the treatment, the initial fat content of milk was determined in accordance with the standard procedure. Then the milk was subjected to multiple treatments in a static type cavitation device, described in detail in [28, 29].

To estimate the homogenization quality of the milk the measuring of the fat phase sizes by the microscope was used along with the microphotography and computer analysis of the obtained data (analysis of the milk assay microphotography). To take them the optical microscope Micromed XS-2610 was used, to which the web-camera was fixed being in its turn connected to the PC. The preparation of the milk assays was as follows: assays mixing; selection and solution of the assay by the distilled water in 1 to 40 proportion (three solutions were prepared from each assay and two compounds from each solution); spreading it on the polished glass; keeping the assay for 20-30 min at the room temperature; taking photography. Multiplication ratio was 640 times while taking the photo of $10,4 \times 8,0 \mathrm{~cm}$ size (the cameracapacity being $640 \times 480$ pickcels). While investigating the number of fat balls and their diameters were calculated being identified by the microscope. The calculation was performed taking advantage of the software IBAS. It makes possible to determine and analise more than 20 parametersof every fat ball: image projection surface, its maximum and minimum diameters, the diameter similar to that of circle cross-section in particular, as well as different parameters of image field, number of all particles, percentage of the field filling by the particles, etc. The size of intervals of the fat ball groups was assumed to be $0,5 \mathrm{mkm}$. The number of intervals $\mathrm{n}$ were calculated approximately due to the expression $n=\sqrt{N}$ where $N$ is the number of calculated fat balls. The mean diameter of the fat balls, mean-quadratic deviation and coefficient of variation were determined.

\subsection{Preparation of fat emulsions}

In the laboratory, emulsions with a $35 \%$ fat phase concentration were used. For its preparation reconstituted skimmed milk powder and as a fat phase, pure milk fat and milk fat substitutes were used: "Olmix 100 AK" produced by the Kyiv Margarine Plant, "Fettimilk 02AK" produced by Zaporizhzhya Fat-and-Food Complex and "Delikon ZTL No. 1" produced by Modified Fats Plant in Kirovohrad (Kropyvnytskyi). Emulsifiers: distilled monoglycerides and soy lecithin (in a ratio of 3:1) were used in the studies, which were put into the emulsion fat phase at $65^{\circ}$ and $50^{\circ} \mathrm{C}$, respectively. The experiments were repeated 2-3 times. Emulsions were evaluated according to the degree of destabilization, resistance and dispersion of the fat phase. Natural cream of the appropriate fat content was taken as a reference standard. 


\subsection{Product and methods for investigation of the rheological parameters of the cream cheese mass}

The main components of the product were cream chees, water and flavours, the content of jelly substances being $1 \% \ldots 5 \%$ of the total mixture mass. The content of cheese mass is $70,1 \%$, water $-17,7 \%$, flavours $-12,2 \%$, unified rheological coefficient $-3,193$.

\subsection{Temperature measurement}

To measure the temperature the method of the contact temperature measuring was used using the C-K thermocouples $-50 \div 0 \div 800 \mathrm{C}$, connected with the dsgital image potentiometer, while investigating the temperature in the flow of the operating mixture the thermocouples were set technologically correspondingly. The engineering parameters of the C-K termocouples are those of technical State standard 6616-94 "Thermoelectrical transformers. General technological requirements".

\subsection{Vacuum measurement}

The vacuum measurement during the mixture treatment was perfomed by the mechanic vacuum-measuring using the vacuum manometer the measuring limit of the exceeding pressure being $500 \mathrm{KPa}$ of 1 class accuracy: technical State standard 2405-88. The vacuum manometer was connected with the operating camera of the test installation through the tread hole being fixed in the operating camera cap.

\subsection{Analysis of microstructure}

Investigation of the food products microstructure deals with the multiple increase of the prepared assay by the the optical devices while taking the image using the photographic camera. In the case in question the microstructural analysis of cream cheese masses was performed using the light optical microscope Motic (Fischer Bioblock) photovideo camera being integrated. During the experiment the product assay was thin spread on the polished glass by the microbiologocal loop. After that it was dried and being covered by the glass cap it was put under the microscope eyepiece, the scale being 400 -times.

\subsection{Investigation of rheological characteristics}

Rheological characteristics were studied at the "Rheotest 2" stnd. Yield viscosity is from 10-2 to $104 \mathrm{~Pa} \cdot \mathrm{c}$; shearing rate is from 0,1667 till $1458 \mathrm{c}-1$; shearing stress is from 12 till $3000 \mathrm{~Pa}$; temperature is from -30 till $+150^{\circ} \mathrm{C}$. Measument error $\pm 3 \%$ (for the Newtonian liquid). New portion (assay) of the product was taken for each experiment. Data were recorded after the full rotation of the inner cylinder, the product thermostatics being provided. Taking into account the structural-mechanical properties of the investigated product, the rheostat operation mode was that of «a», where «Rheotest 2» engine speed was $1500 \mathrm{rev} / \mathrm{min}$.

The prepared assay of the cream cheese mass was put in the rheostat outer cylinder. Than the sinking of the inner cylinder followed. The measurement of the tangential shearing stress $(\mathrm{Pa})$ was performed at twelve values of the shearing rate gradient within the range of $0,33-145,8 s-1$, the data of the device $\alpha$ measuring scale being recorded at the maximum angular deflection of the device scale pointer.

\subsection{Analysis of rheological characteristics}

While analyzing the rheological characteristics of non-Newtonian liquids the liquidlike systems without the yield shearing stress are specified (the equation of Ostwald-deWaele's, Eyring-Powell, Barteneva-Yermylova etc) and those with the yeild shearing stress (the equation of Shvedov-Bingama, Gershel'-Balkli, Kesson, Shulman, MyxajlovLyxtgejm etc). To describe the rheological characteristics of the cream cheese mass type food products the equation by Ostwald-de-Waele and Herschel Bulkley were used.

The shearing stress $(\mathrm{Pa})$ was calculated due to the formula:

$$
\tau_{r}=Z \cdot \alpha,
$$


where $\quad Z-$ is the cylinder constant, scale Pa/unit;

$\alpha$-are the measuring device scale factors.

The effective viscisity $(\mathrm{Pa} \cdot \mathrm{sec})$ was calculated due to the formula:

$$
\eta_{\text {eff }}=\frac{\tau_{r}}{\gamma}
$$

where $\quad \gamma-$ is the shearing rate gradient, $\mathrm{s}-1$.

According to the measurement results the arithmetical mean values were found, due to which the graphs were plotted and further mathematic processing was carried out. The value of the yield shearing stress and effective viscosity at the unit value of the rate gradient were determined using the methods of methematic analysis taking advantage of the software MS Excel.

Dependence of the shearing stress on the shearing rate for the cream cheese mass can be presented as the Ostwald-de-Waele's dependence:

$$
\tau_{r}=B^{*} \cdot \gamma^{n},
$$

where $\quad \tau_{r}-$ is the shearing sress between the products layers, Pa;

$B^{*}-$ is the viscosity at the unit value of the rate gradient, $\mathrm{Pa} \cdot \mathrm{s}$;

$\gamma-$ is the relative rate gradient being numerically equal to the shearing rate, $s-1$;

$n-$ is the flow index.

To establish the influence of technological and mechanical factors on the rheological characteristics of the "Yagidka" dessert products, experiments were carried out in three stages: 1) under minimal mechanical processing and variable temperature of the product's heating; 2) under constant temperature and variable power of mechanical processing; 3) under the apparatus nominal mode. Only the average values of the studied parameters were analyzed. The value of effective viscosity at a single value of the velocity gradient, as well as the boundary shear stress, was found by mathematical analysis methods using the OpenOffice 2.0 application package.

\section{Results}

\subsection{Milk fat homogenization features in milk}

Experimental results on the change in the coefficient of milk homogenization depending on the treatment cycles number in the static cavitational device (Figure 1) at various temperature values are shown in Figure 2. It was established that at 6 cycles of treatment the best result of homogenization $-78 \%$ was obtained at the temperature of $72^{\circ} \mathrm{C}$.

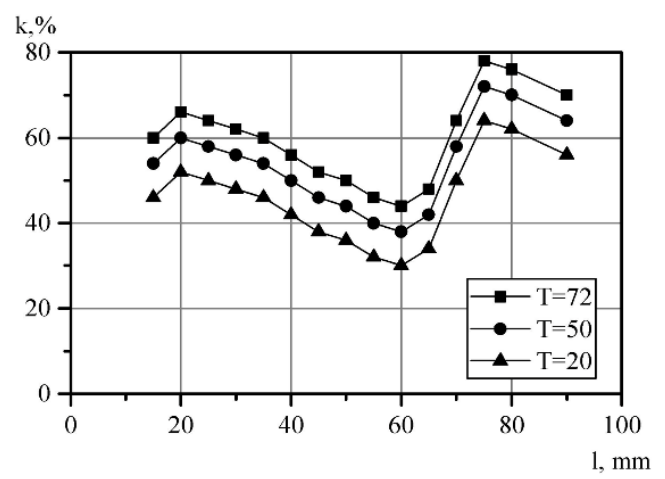

Figure 1. Milk homogenization coefficient versus the number of processing cycles at different temperature values curve: $1-20^{\circ} \mathrm{C}, 2-50^{\circ} \mathrm{C}, 3-72^{\circ} \mathrm{C}$. 


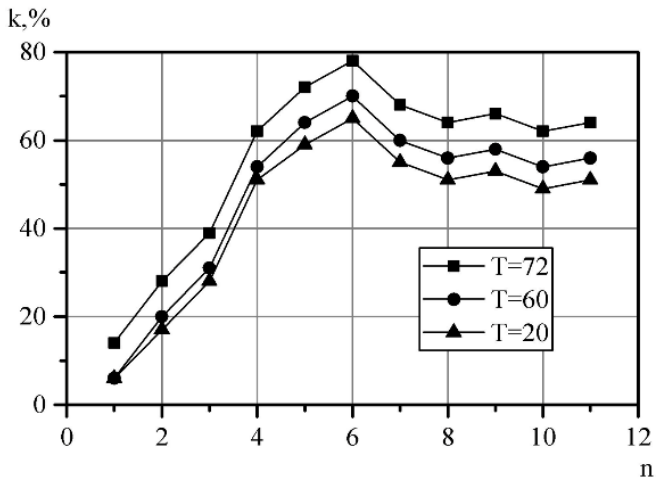

Figure 2. Milk homogenization coefficient versus the distance between the nozzle and the buffle curve: $1-20^{\circ} \mathrm{C}, 2-50^{\circ} \mathrm{C}, 3-72^{\circ} \mathrm{C}$.

The greater efficiency of treatment at the temperature of $72^{\circ} \mathrm{C}$ can be explained by a decrease in the viscosity of fat particles, the softening of their lipid membranes, and also by the fact that there is a partial thermal deaeration of the product.

During the research it was established that the effective multiplicity of milk processing with fat content of $4 \%$ is 6 times, and the homogenization efficiency is almost identical at the temperature of $20^{\circ} \mathrm{C}$ and $50^{\circ} \mathrm{C}$ and a cavitation stage of $2.5 \ldots 3.0(65 \%$ and $70 \%$ respectively). In this case, this occurs due to a decrease in the viscosity of fat particles, softening of their lipid membranes and melting of even the most refractory fractions of milk fat. This creates a system "liquid-liquid", where partial thermal deaeration of the product takes place, in which the effectiveness of hydromechanical influence of cavitation increases.

Figure 2 shows the dependence of the milk homogenization coefficient on the distance between the narrowed area and the buffle in the six-fold treatment in the flow type apparatus, with milk temperature of $72^{\circ} \mathrm{C}$. The curve of the coefficient of homogenization versus the distance between the nozzle and the buffle shows that there is an optimal distance from the nozzle to the buffle, at which the degree of homogenization is the highest. At a distance of $20 \mathrm{~mm}$ and six-fold milk processing in the cavitational apparatus, the value of the coefficient of homogenization is within the range of $50 \%-70 \%$. According to the received plot, with an increase in the distance between the nozzle and the buffle up to $60 \mathrm{~mm}$ and the constant speed of the fluid and temperature, the homogenization rate decreases to $30-42 \%$. With further increase in distance, an increase in the coefficient of homogenization up to a critical value of $78 \%$ is observed, which corresponds to a distance of $75 \mathrm{~mm}$ from the nozzle to the buffle.

On the other hand, the intensity of the cavitation effect increases at relatively low temperatures, which provide a reduced elasticity of water vapor. In this case, the influence of cavitation occurs in a medium containing a liquid with distributed in it solid fat particles, that is, in the system "liquid-solid body" The obtained effect resulted from the turbulent fluctuations impact spheric waves, cumulation spray, when cavitation bubbles are crashed. The homogenization effect mechanism is presented on Table.1.

Table 1. Mechanism of the milk fat disperasion.

Drops turbulent dispersion: isotropic and viscous. The mechanism of dispersion isotropic turbulence is followed by the stress fluctuations caused by the microvorticity. Under the viscous mechanism it is caused by the shearing stress of more sufficient vor-
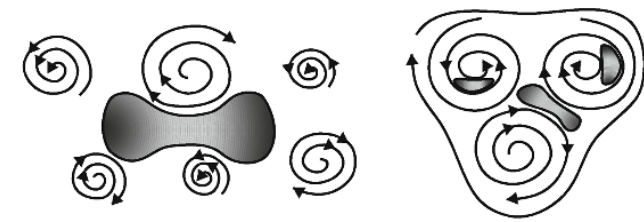
ticity.

In the cavitation zone fluctation cavitation bubbles arise, which, being collided with the dispersion phase drops, are buffeting.

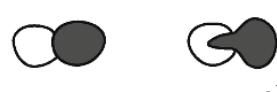

a) 


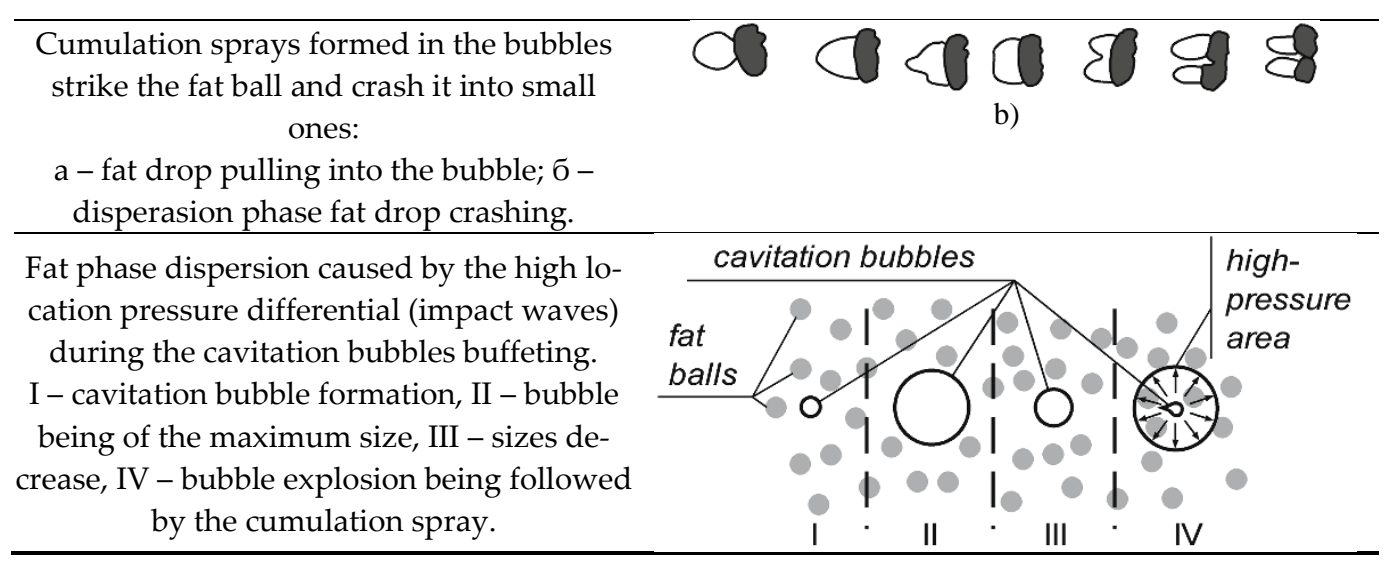

Changes in the fractional composition of the fat balls according to sizes after homogenization in the cavitation device as compared with the valve homogenization is presented in Figure 3, microphotography of the milk assays - in Figure 4.

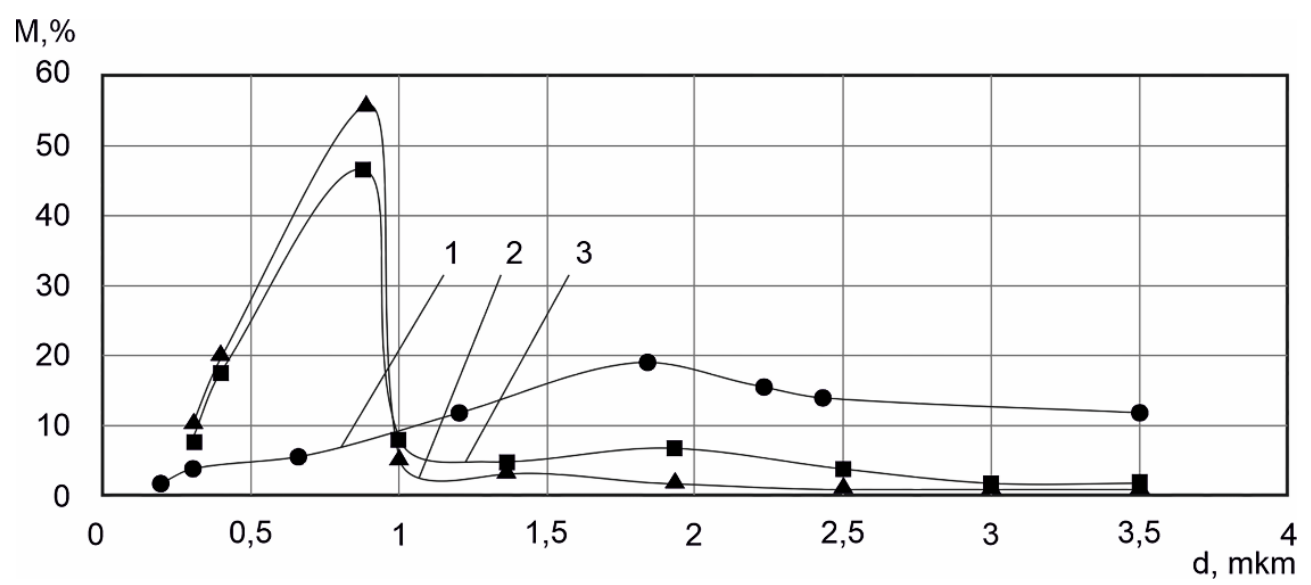

Figure 3. Distribution of the fat balls according to the sizes: 1-non-homogenized milk; 2- milk after the valve homogenization; 3 - milk after treatment in the cavitation device.

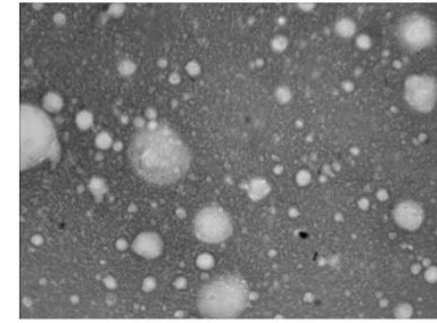

(a)

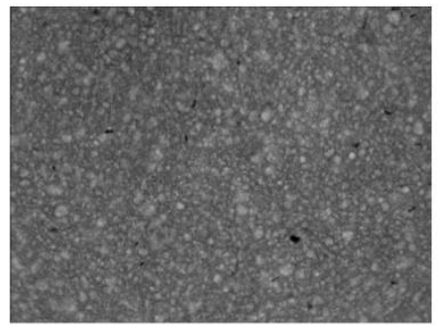

(b)

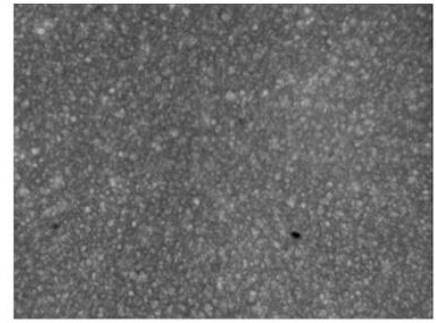

(c)

Figure 4. Microphotography of milk: (a)-non-homogenized; (b)- after the valve homogenization; (c)- after treatment in the cavitation device.

Milk parameters before homogenization: fat balls mean diameter dcep $=2,6 \mathrm{mkm}$, dispersion $\sigma=1,44$, coefficient of variation (the portion of the feature scattering relatively the mean one) $\mathrm{V}=67 \%$. After the valve homogenization and corresponding impulse homogenization these data are as follows: dmean $=0,96 \mathrm{mkm}$ and $0,9 \mathrm{mkm}, \sigma \sigma=0,50$ and $0,46, \mathrm{~V}=51$ and $56 \%$. Mean diameter of the fat balls while treatment in the cavitation device was decreased by $6 \%$ as compared with the valve one. Besides, dispersion value was decreased also, which, in its turn, testifies that the homogenization in cavitation device provides stability of the milk fat phase after homogenization.

The effect of the distance between the nozzle and the buffle on the homogenization rate can be explained in the following way. When changing the position of the obstacle 
relative to the nozzle, the geometry and volume of gas cavities have changed. At a distance of 20 and $100 \mathrm{~mm}$ the most active formation of small size caverns and their rapid collapse right after an obstacle without reaching the next level have been observed. When increasing the distances, the size of the cavity increases, and the intensity of their formation decreases, which leads to a decrease in the homogenization coefficient.

These results have been proved by the data given in the papers [24-27]. The authors note that the processing of milk and cream in cavitation devices affects the size of fat particles, the relative viscosity of the product, in particular, the diameter of the fat particles is reduced up to 1.0-1.5 microns, relative product viscosity increases. In work [24] the effect of hydrodynamic cavitation on the preparation of whole milk substitutes with the size of fat particles of 0.5-2 microns was under study. The efficiency of cavitation treatment is explained by the high rate of flow in the fluid processes that determine the mechanical model of cavitation action. These are: the creation of intense fields of pulsating pressures (10 - $10 \mathrm{~atm})$ and waves of discharge; formation of cumulative micro streams; formation of turbulent zones in flow right after buffle with swirling and collapsing microbubbles; phase transitions occurring on the surface of cavitation bubbles; temperature fluctuations in the vapor-gas content of cavities at their collapse.

\subsection{Patterns of fat dispersions formation under discrete-impulse energy supply}

The results of fat dispersions formation patterns in rotary device have proved that the process of fats emulsification in milk plasma is cyclical in its nature (Figure 5, 6). After obtaining a dispersion with an average size of fat bubbles ( $<4$ microns), processes of dispersion and aggregation of fat bubbles, accompanied by an increase and decrease in the stability of the emulsion, occur alternately. The most effective emulsification takes place at a rotor speed of a dispersing device of $3000 \mathrm{rpm}$. (Figure 6). In this case, after 1,5 min. of treatment a fine-dispersed (fat particles average size $<2$ microns) and durable emulsion (a degree of destabilization of about $30 \%$ ) has been formed. The emulsion close to the described characteristics has been obtained also at the intensity of the corresponding rotation speed of the rotor processing of the dispersing device $2500 \mathrm{rpm}$. The increase of the intensity of emulsion treatment in the rotary device leads to the power increase. So at a speed of $1500 \mathrm{rpm}$. it is about 60 Watts, and at $3000 \mathrm{rpm}$. reaches 150-200 Watts. 


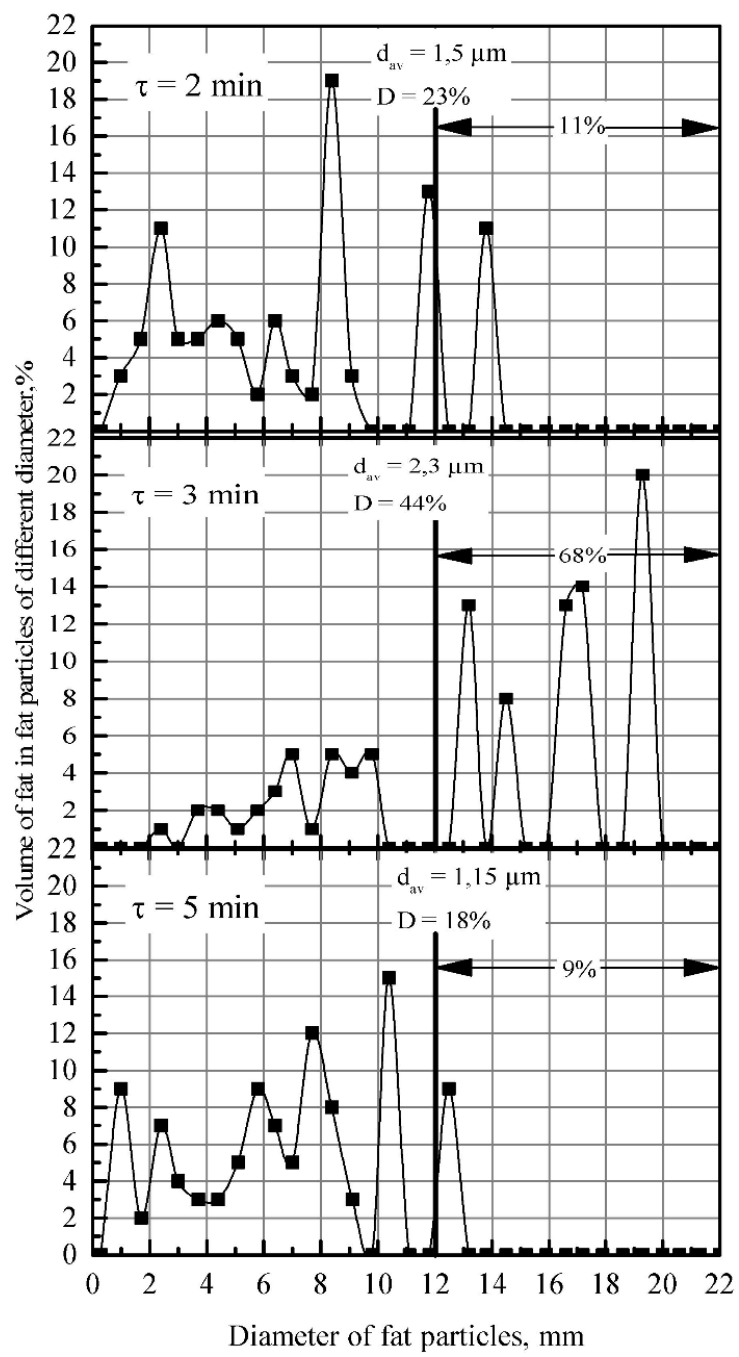

Figure 5. Varied granulometric composition of fat emulsion during processing in the emulsifying device ( $\mathrm{n}=2500 \mathrm{rpm}$ ). 


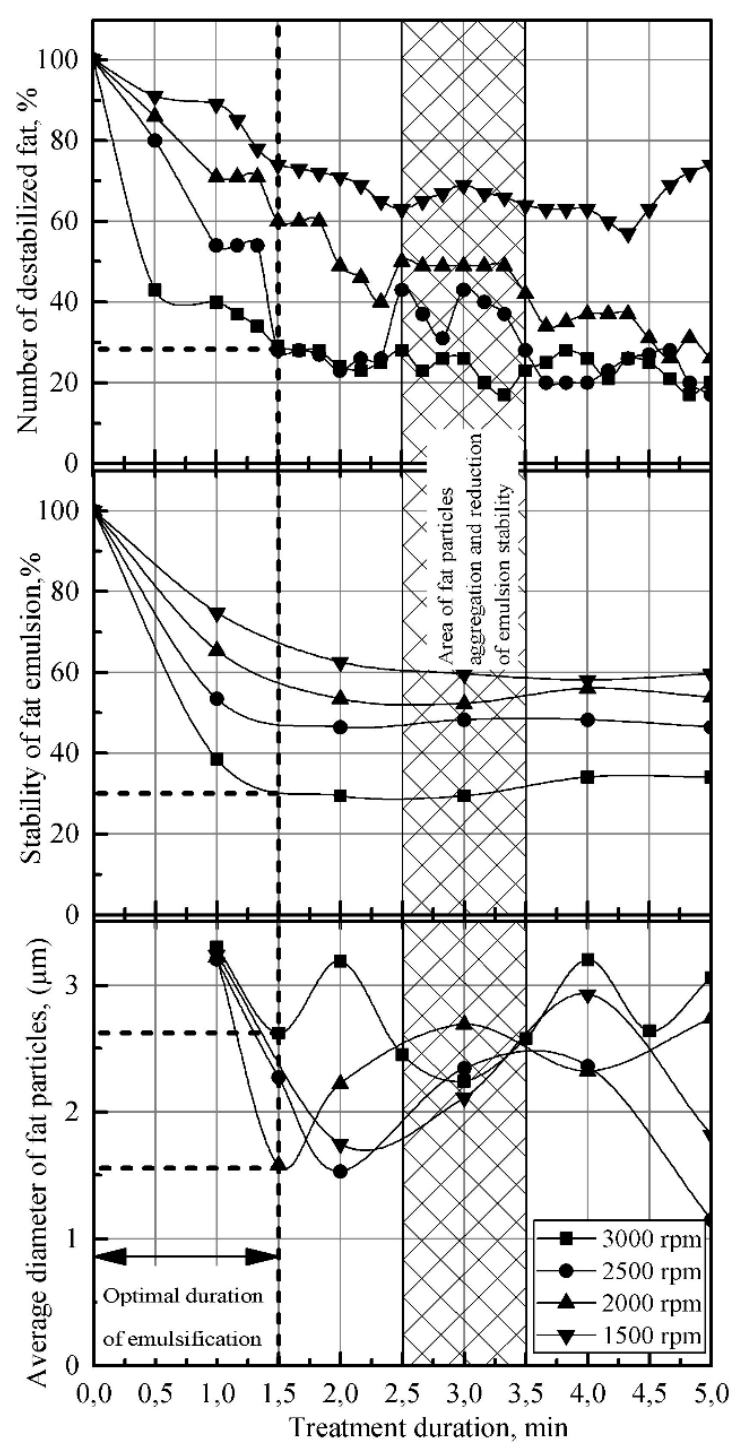

Figure 6. Varied degree of dispersion and stability of fat emulsion in the process of treatment in the emulsifying device.

It is established that to obtain an emulsion with dispersion and stability close to natural creams, it is preferable to do emulsification without vacuuming at a temperature 50$70^{\circ} \mathrm{C}$. As the concentration of surfactant decreases, optimal treatment duration is reduced. However, the excess of the emulsifier does not increase the stability of the emulsion. In this case, the optimal concentration of surfactant is $0.6 \%$ (distilled monoglycerid+ lecithin in the ratio of $3: 1$ ), which ensures the production of a technologically stable emulsion of $35 \%$ fat, using a rotary type device (Figure 7 ).

The results have proved that the use of the fat phase of different types of fats does not significantly affect the emulsification processes (Figure 8). This also applies to power and energy consumption during emulsification. At the same time, the increase in the concentration of the fat phase slows down the formation of the emulsion, as it increases the degree of its destabilization and, accordingly, the optimal duration of emulsification. This, as well as the increase of the viscosity of the emulsion with an increase in its fat content, leads to the cost increase of power and energy for emulsification, which increases with increasing fat content from 3.5 to $35 \%$ in $\approx 1.6$ times.

Taking into account all the above mentioned, we can argue that the optimum parameters for obtaining an emulsion with a fat content of $35 \%$ (with dispersion and stability close to natural creams) in a rotary type device, regardless of the type of fat phase used, at the concentration of surfactant- $0.6 \%$ are the following: temperature of emulsification $50-70^{\circ} \mathrm{C}$, processing power $150-200$ Watts, with a rotor speed of the emulsifying device of 
$3000 \mathrm{rpm}$; processing time 1-1.25 min.; the energy consumption in this case will be 2-3.5 $\mathrm{kJ} / \mathrm{kg}$.

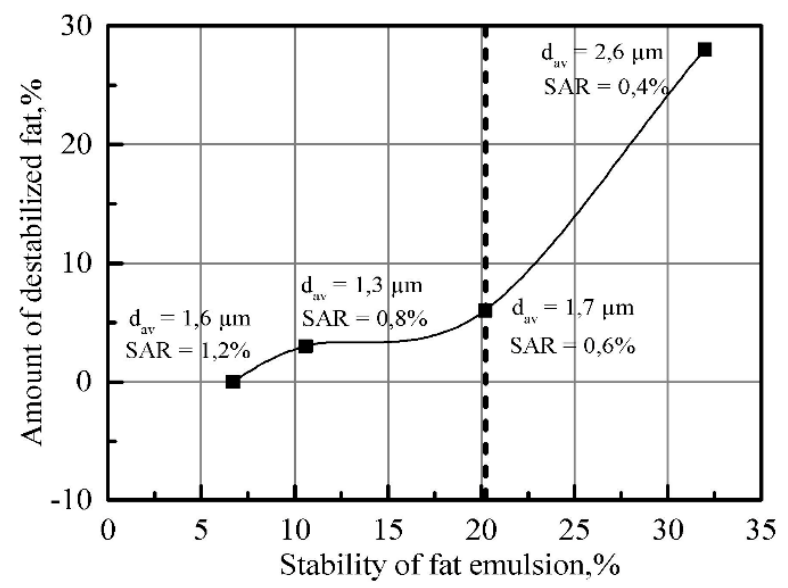

Figure 7. Variations of the dispersion degree and fat emulsion stability depending on the concentration of surfactant ${ }^{*}$ - concentration of surfactant, $\%$ : monoglycerid+ lecithin in the ratio of $3: 1 ; \tau$ $=1.5 \mathrm{~min}$.).

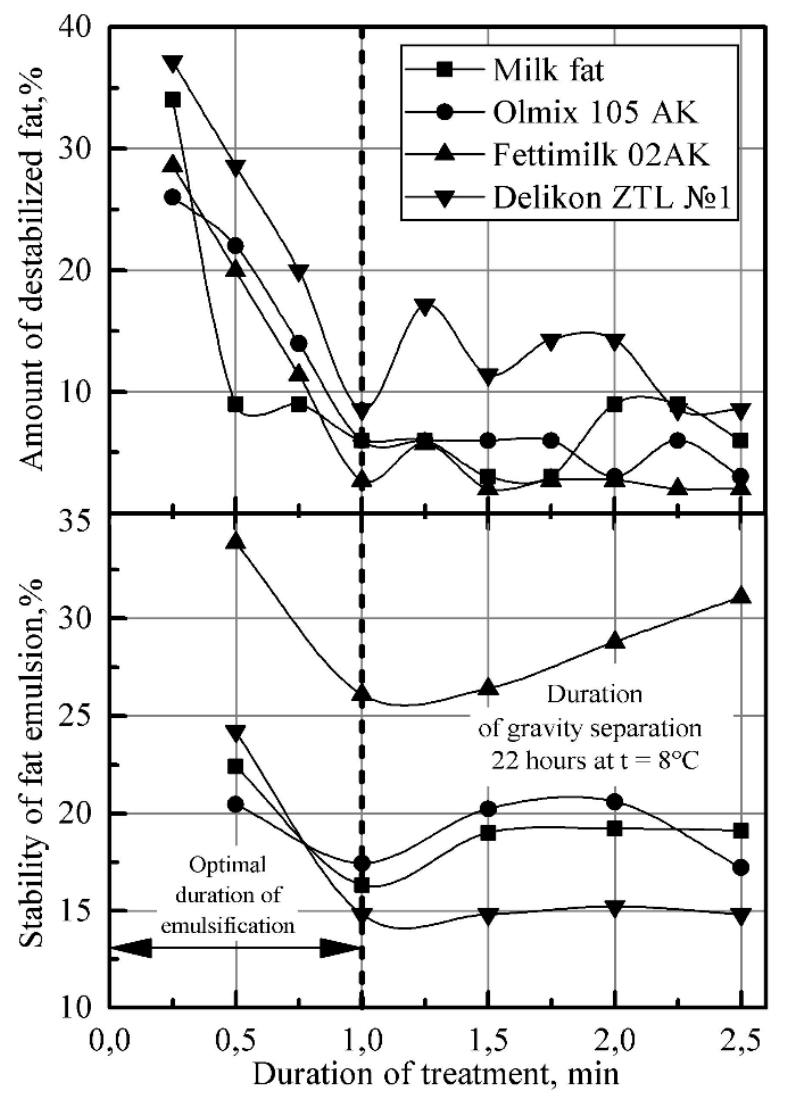

Figure 8. Varied fat emulsion stability during treatment in the emulsifying device (concentration of surfactant- $0.6 \%$ : monoglycerid +lecithin in the ratio of $3: 1$ ).

\subsection{The rheological characteristics variations of cream cheese masses}

The dependence of the viscosity of the dessert mass on the shear rate for the temperature range under study indicates an increase in the shear rate and a decrease in the effective viscosity (Figure 9, 10). 


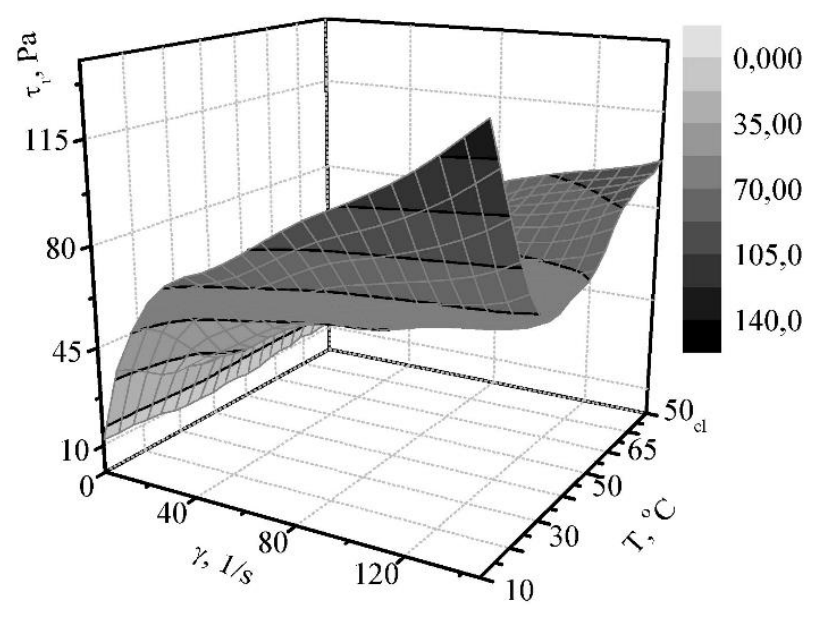

Figure 9. The dependence of shear rate for the product "Yagidka" on the shear rate and time under minimal mechanical treatment.

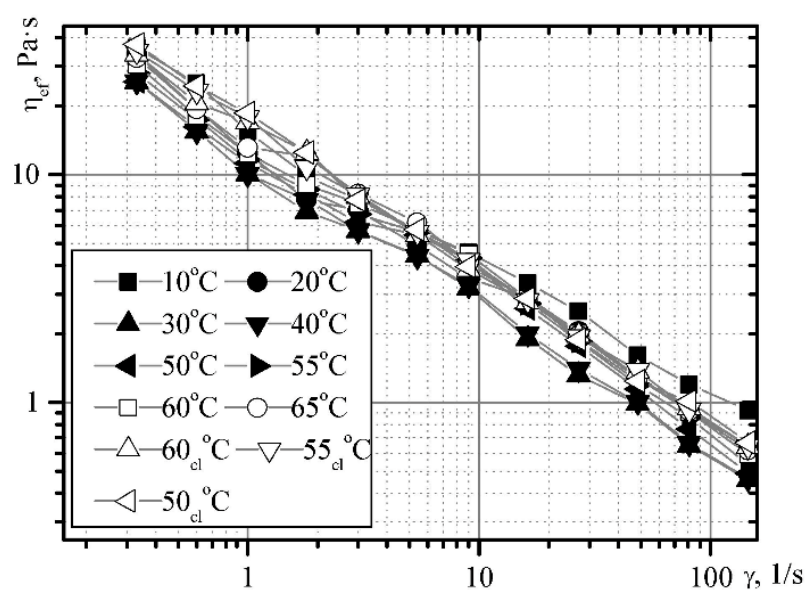

Figure 10. The dependence of the effective viscosity on the shear rate for the product "Yagidka" under minimal mechanical treatment.

With an increase in temperature from $10^{\circ}$ to $30^{\circ} \mathrm{C}$, the viscosity and shear rate decrease and remain approximately constant within the temperature range of $30^{\circ}-50^{\circ} \mathrm{C}$ and with increasing temperature they increase. This happens due to the structure of the thickener (pectin), for which the temperature of gelling is in the range of $52^{\circ} \ldots 55^{\circ} \mathrm{C}$. The boundary shear rate allows to take into account the dependence of Herschel-Bulkley.

$$
\tau=\tau_{0}+\mathrm{B} \cdot \gamma^{n}
$$

where $\quad \tau_{0}-$ is the boundary shear rate, Pa.

The influence pattern of the heating temperature on the effective viscosity is maintained. The value of the rate of structure destruction, which varies within fairly narrow limits, indicates the correctness of the experiment. For an intact structure at $10^{\circ} \mathrm{C}$ - at the initial stage of the process - the dependence has the following form:

$$
\tau_{r}=9,1+11,5 \cdot \gamma^{0,475} .
$$

At $65^{\circ} \mathrm{C}$ (in end of the heating process), the dependence has the following form:

$$
\tau_{r}=9,3+9,6 \cdot \gamma^{0,429}
$$

Under cooling to $50^{\circ} \mathrm{C}$ :

$$
\tau_{r}=9,6+10,0 \cdot \gamma^{0,434}
$$


The dependences graphs of the boundary shear rate and effective viscosity on the duration of machanical treatment at a temperature of $10^{\circ} \mathrm{C}$ are shown in Figure 11, 12 .

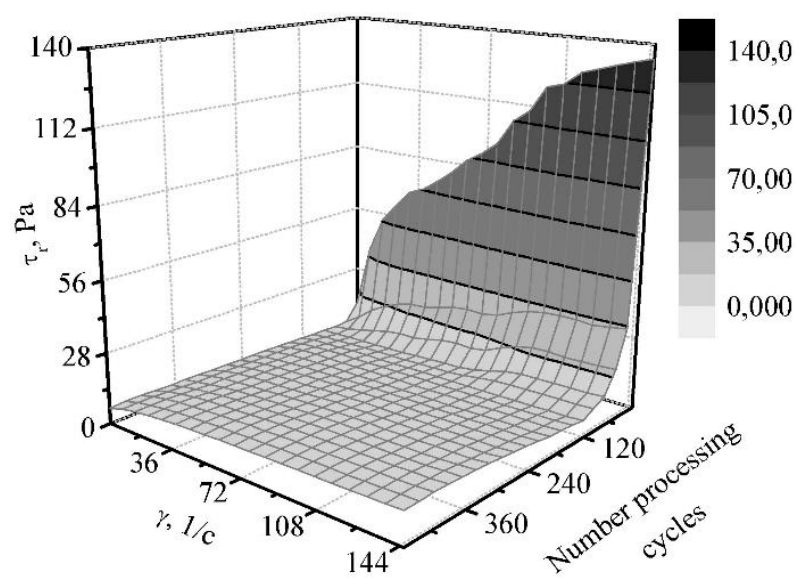

Figure 11. The dependence of the shear rate for the product "Yagidka" on the shear rate in the emulsifier at a temperature of $10^{\circ} \mathrm{C}$.

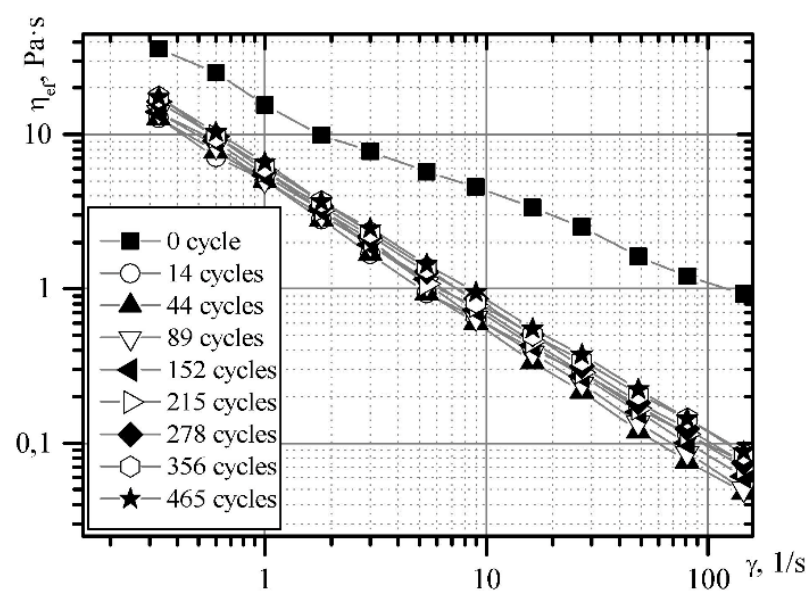

Figure 12. The dependence curve of the effective viscosity for the product "Yagidka" during the treatment in an emulsifier at a temperature of $10^{\circ} \mathrm{C}$.

Under the condition of no mechanical processing with increasing angular deformation rate, the boundary shear rate increases. At the same time, the structure destruction is observed in the first cycles of processing. Hereafter, the boundary shear rate, as well as other rheological characteristics, remains almost constant. However, when the processing time of cream cheese mass exceeds 260 cycles of mechanical treatment, an increase in the boundary shear rate is observed and the effective viscosity slightly increases. This happens due to the processes that take place at the micro level, in particular the phenomena of cavitation, local heating, and the swelling of the structure-forming agent.

Ostwald-de Waele rheological equation for the mass at the initial stage of the process has the form:

$$
\tau_{r}=12,5 \cdot \gamma^{0,472}
$$

Rheological equations in the end of the process in the form of Ostwald-de-Waele dependence

$$
\tau_{r}=5,1 \cdot \gamma^{0,195}
$$

Herschel Bulkley -

$$
\tau_{r}=5,5+1,16 \cdot \gamma^{0,370}
$$


It should be noted that the destruction of the mass structure takes place during the first 20 cycles of product circulation, and the mass heating takes place in a completely destroyed structure. As a result of the analysis of the product movement process in the rotor-stator pair, it is evident that not the whole transported product, but only its part (Figure 13), is the subject to mechanical grinding.

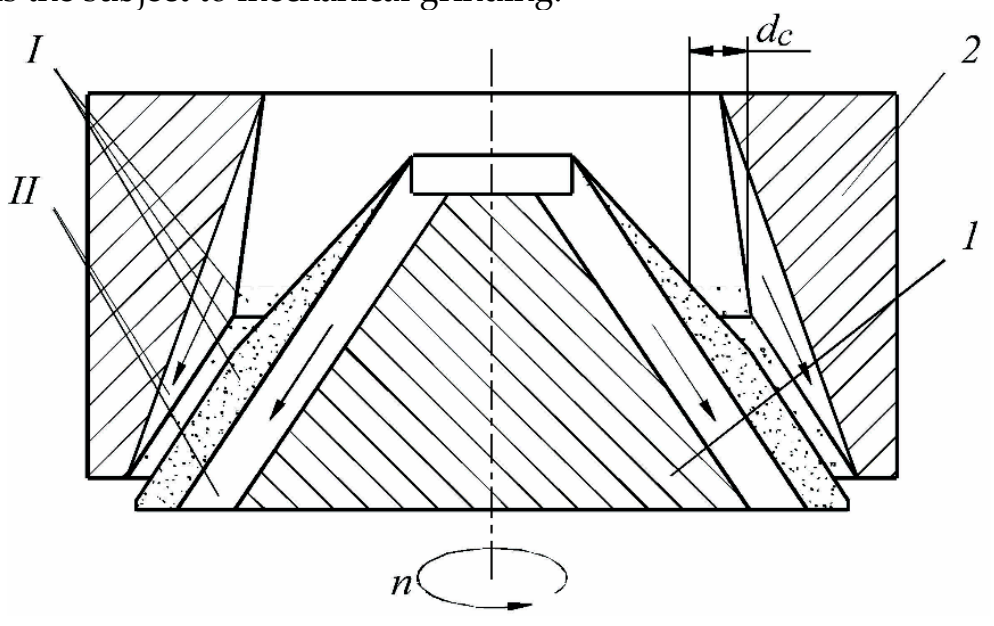

Figure 13. The scheme of movement of cream cheese mass in the channels of the rotary-vortex emulsor

1-rotor, 2-stator; I-a zone of transportation and mechanical treatment of cream cheese mass, II-a zone of transportation without mechanical processing of cream cheese mass.

One can distinguish zones in which the nature of the mass movement will be different: zone I is the area of the most active mechanical effect on the product (the width of this zone at its beginning in the direction of product movement $d_{c}$ corresponds to the size of the transported mass particles with adjacent layers), in which occurs the grinding between a rotor and a stator at the expense of a large velocity gradient and at low stress on a cut of cream cheese masses; in zone II occurs mainly the transportation of the product without its mechanical processing, neglecting some mass exchange between zone I and II. The product, captured by the grooves, will also be destroyed.

Let's denote the volume of the loaded mass by $V_{0}$. Then the volume of mechanically treated product after one cycle of passing through a pair of rotor stator will be $\psi \cdot V_{0}$. Then the volume of the unprocessed product will be $(1-\psi) \cdot V_{0}$.

Due to the peculiarities of the geometric parameters of the mass flow channels, we have assumed that the coefficient of proportionality $\psi$ corresponds to the ratio of the total area of zone I to the total area of zones I and II in the lower part of the rotor-stator pair.

Let's analyze several stages of product mechanical processing during its movement in the circulation circuit of the apparatus.

Let's denote the running volume of the treated material by $V_{b}$, the running cycle by $i$, and the multiplicity of processing at this stage by $\kappa$. Then the dependence of the multiplicity of processing of running volume will have the form:

$$
V_{b}=\Delta \cdot \psi^{\kappa} \cdot(1-\psi)^{i-\kappa} \cdot V_{0}
$$

where $\quad \Delta$ - the numerical coefficient.

In the general case, it has the form:

$$
\Delta=\Delta\left(\begin{array}{l}
\kappa \\
i
\end{array}\right)=\frac{i !}{(i-\kappa) ! \kappa !} .
$$

Then the formula (9) can be rewritten as the following one: 


$$
V_{b}\left(\begin{array}{c}
\kappa \\
i
\end{array}\right)=\frac{i !}{(i-\kappa) ! \cdot \kappa !} \cdot \psi^{\kappa} \cdot(1-\psi)^{i-\kappa} \cdot V_{0} \cdot
$$

Using the dependence (10), it is possible to estimate the level of mechanical processing of the loaded product. For example, in order to provide the relevant technological qualitative indicators, it is necessary to ensure the multiplicity of machanical treatment $r$. Then for quantitative estimation of mechanical processing at an arbitrary stage of processing of raw materials $V_{r}$ it is necessary to find the sum of all parts of raw materials except for those which are processed less than $r$ times:

$$
V_{r}=V_{0} \cdot \sum_{\kappa=r}^{i} \frac{i !}{(i-\kappa) ! \cdot \kappa !} \cdot \psi^{\kappa} \cdot(1-\psi)^{i-\kappa}
$$

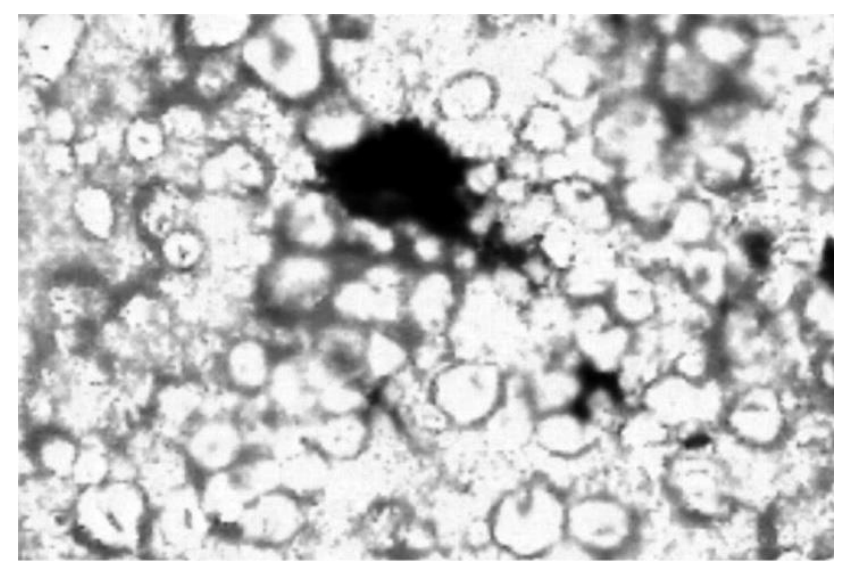

(a)

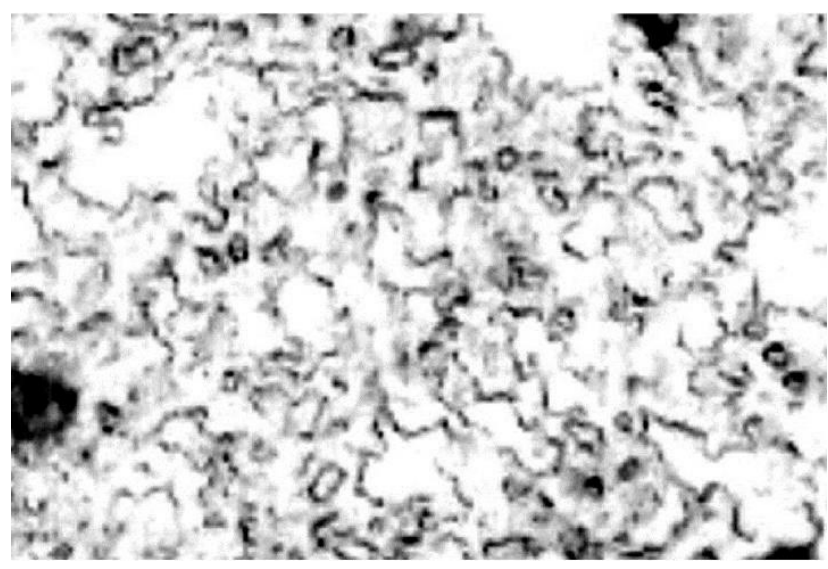

(b)

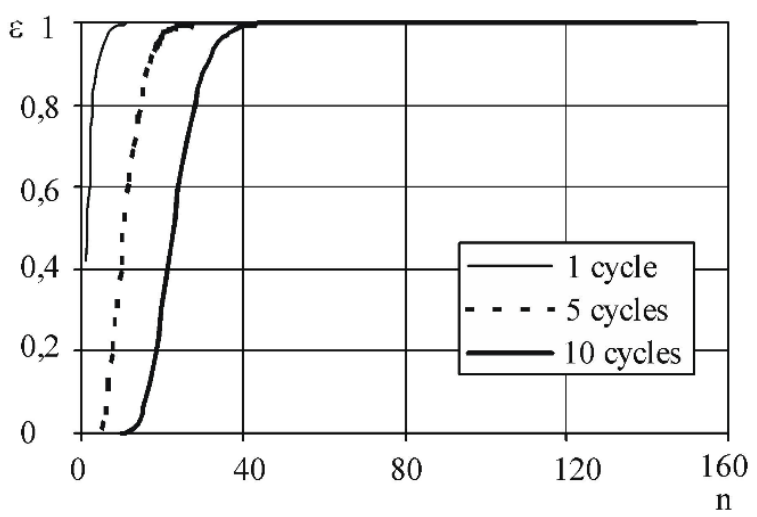

(c)

Figure 14. Microstructure of the cream cheese mass "Yagidka": (a) the microstructure of the cream cheese mass before treatment; (b) the microstructure of the cream cheese mass after 45 cycles of treatment; (c) the level of mechanical treatment of the product depending on the processing cycles.

The ratio of the sufficiently treated raw material $V_{r}$ to its initial quantity $V_{0}$ is proposed to be considered as an index (coefficient) of mechanical treatment in cyclical apparatus:

$$
\xi=\frac{V_{r}}{V_{0}}=\frac{V_{0} \cdot \sum_{\kappa=r}^{i} \frac{i !}{(i-\kappa) ! \cdot \kappa !} \cdot \psi^{\kappa} \cdot(1-\psi)^{i-\kappa}}{V_{0}}=\sum_{\kappa=r}^{i} \frac{i !}{(i-\kappa) ! \cdot \kappa !} \cdot \psi^{\kappa} \cdot(1-\psi)^{i-\kappa} .
$$

Let's assume that tenfold mechanical processing is sufficient for proper maintenance of the finished product structure. From the dependence (Figure 14c), according to the for- 
mula (10), it is evident that a guaranteed ten-fold processing of the product will be provided at 45 cycles of circulation of the product through a rotor-stator pair. The microstructure analysis of the product "Yagidka", performed by the optical microscope Motic (Fischer Bioblock) with an integrated photo-video camera (Fig $4.16 \mathrm{a}, \mathrm{b}$ ) at the initial moment of time and at a time corresponding to 45 cycles of mechanical processing, has showed that such duration of mechanical processing is quite enough to ensure the formation of the structure of the product and provide an equal distribution of its components in the volume. The maximum particle size was $0.2 \mathrm{~mm}$. Thus, it can be noted that the required machanical level of treatment is provided long before the completion of the process cycle.

\section{Conclusions}

The study under discussion has found that the effective freof milk processing with a fat content of $4 \%$ in a three-stage apparatus corresponds to 2 times, and the homogenization efficiency is almost identical at a temperature of $20^{\circ} \mathrm{C}$ and $50^{\circ} \mathrm{C}$ and a cavitation stage of $2.5,3.5$ ( $80 \%$ and $86 \%$ correspondingly). In this case, this can be explained by the decrease in the viscosity of fat particles, softening of their lipid membranes and melting of even the most refractory fractions of milk fat. This creates a system of "liquid-liquid", there is a partial thermal deaeration of the product, in which the effectiveness of hydromechanical influence of cavitation increases. On the other hand, the intensity of the cavitation effect increases at relatively low temperatures, which provide a reduced elasticity of water vapor. In this case, the influence of cavitation occurs in a medium containing a liquid with distributed in it solid fat particles, that is, in the system "liquid-solid body".

According to the obtained data on the patterns of formation of fat dispersions in the rotary type device, it has been shown that the emulsification of fats in the milk plasma by a rotary type device has a cyclic nature: after obtaining dispersions with an average size of fat particles with the size $<4$ microns, their dispersion and aggregation resulted in an increase and decrease in the stability of emulsions respectively. It was found that the increase in the mass fraction of the fat phase in the mixture (from 3.5 to $35 \%$ ) slows down the formation of an emulsion of $\mathrm{f} / \mathrm{w}$ type, as this increases the degree of its destabilization (from 0 to $30 \%$ ) and, consequently, the duration of effective emulsification (from 30 to $90 \mathrm{~s}$ ). It has been established that the use of the fatty phase of various fats does not significantly affect the emulsification either by the efficiency of the dispersion or the rational duration of the treatment.

The results of the study of the main rheological characteristics of cream cheese mass (effective viscosity, boundary shear rate, rate of structure destruction) during the processing have proved that the destruction of the mass structure takes place during the first 20 cycles of product circulation, and the mass heating takes place in a completely destroyed structure. According to the results of the research, mathematical dependences of the consumed power on the rheological characteristics of the mass have been suggested. The influence of the parameters of the rotor-vortex emulsor on the power consumption has been studied. It was found that the assured ten-fold processing of the product, where the required microstructure of the cream cheese mass has been provided, takes place at 45 cycles of circulation of the product through a rotor-stator pair, which is $2 \ldots 3$ times less than the duration of the main technological cycle.

\section{Patents}

Author Contributions: Conceptualization, Tetiana Vitenko and Viktor Voroshchuk; methodology, Sergiy Narizhnyy; software, Viktor Voroshchuk; validation, Tetiana Vitenko, Viktor Voroshchuk and Sergiy Narizhnyy; formal analysis, Olha Snizhko; investigation, Olha Snizhko; resources, Paweł Droździel; data curation, Tetiana Vitenko; writing-original draft preparation, Tetiana Vitenko; writing - review and editing, Paweł Droździel; visualization, Olha Snizhko; supervision, Paweł Droździel; project administration, Tetiana Vitenko; funding acquisition, Paweł Droździel. All authors have read and agreed to the published version of the manuscript. 


\section{References}

1. Dolinsky A. A., Ivanitsky G.K. The principle of discrete-pulse energy input - new approach to the development of efficient power-saving technologies // Ann. Review of Heat Transfer. N. Y. Wallingford (UK). Begell House Inc. 2003. P.47-83.

2. Dolinsky A. A., Ivanitsky G.K. Use of discrete-pulse energy input in various production processes // Proc. International Conf. on Transport Phenomena Science and Technology. Beijing (China). Higher Education Press. 1992. P. 89-100.

3. Costa, A. L. R., Gomes, A., Andrade, C. C. P. de, \& Cunha, R. L. (2017). Emulsifier functionality and process engineering: Progress and challenges. Food Hydrocolloids, 68, P. 69-80.

4. Akhtar, M., Murray, B. S., \& Dowu, S. (2014). A novel continuous process for making mayonnaise and salad cream using the spinning disc reactor: Effect of heat treatment. Food Hydrocolloids, 42, P. 223-228.

5. Santana, R. C., Perrechil, F. A., \& Cunha, R. L. (2013). High- and Low-Energy Emulsifications for Food Applications: A Focus on Process Parameters. Food Engineering Reviews, 5(2), P. 107-121.

6. Yang, Y., Marshall-Breton, C., Leser, M. E., Sher, A. A., \& McClements, D. J. (2012). Fabrication of ultrafine edible emulsions: Comparison of high-energy and low-energy homogenization methods. Food Hydrocolloids, 29(2), P. $398-406$.

7. Vial, C., Stiriba, Y., \& Trad, Z. (2014). CFD simulation of flow and mixing in-inline rotor-stator mixers with complex fluids. The Canadian Journal of Chemical Engineering, 93(2), P. 391-403.

8. Dolinsky A. A., Ivanitsky G.K. Theoretical justification of discrete-pulse energy input principle. I. The model of the dynamics of single vapor bubble // Heat-process engineering. 1995. Vol. 17, № 5. P. 28.

9. Lugovskoy A. F., Chukhraev N.V. Ultrasonic cavitation in modern technologies. Kyiv, Publishing and printing center "Kyiv University", 2007. 244 p.

10. Vitenko T.M., Humnitsky Ya.M. The effect of the vapor-gas phase on the kinetics of dissolution under cavitation conditions // East-European Journal of Advanced Technology. Kharkiv. 2009. Vol. 3/6 (39), P. 4-8.

11. Hinze J.O. Fundamentals of the hydrodynamic mechanism of splitting in dispersion processes // A.I.Ch.E.Journ.-1955. №3. P.289-295.

12. Clark N. Drop breakup in a turbulent flow. II.Experiments in a small mixing vessels // Ibid. 1988. №3. P.683-692.

13. Young C.H., Korchinsky W.J. Modelling Drop-side mass transfer in agitated polydispersed liquid-liquid systems // Ibid. 1989. №10. P.2355-2361.

14. Hesketh R.P., Etchells A.W., Russel T.W. Bubble breakage in pipeline flow // Ibid. 1991. №1. P.1-10.

15. Lagisetty J.S., Das T.K., Kumar R., Gandhi K. S. Breakage of viscous and non-Newtonian drops in stirred dispersions // Ibid. 1986. №1. P.65-72.

16. Jares J., Prochazka J. Break-up of droplets in Karr reciproting plate extraction column // Chem. Engng. Sci. 1987. №2. P. 283-292.

17. Dolinsky A.A, Ivanitsky G.K. Heat and mass transfer and hydrodynamics in vapor-liquid media. Thermophysical fundamentals of discrete-impulse energy input // Monograph. Kyiv, Naukova Dumka, 2008. 382 p

18. Basok B., Dolinsky A., Ivanitsky G., Ryzhkova T. Dynamics of single droplets moving throught liquid media // Book of Abstracts of the International Symposium on Liquid-Liquid Two-Phase Flow and Transport Phenomena. Antalya (Turkey). 1997. Session 3. P. 26-28.

19. Suslick K.S. Sonochemistry //In Kirk-Othmer Enciclopedia of Cemical Technology; 4th edition. New York, John Wiley\&sons, Inc., 1996. P.516.

20. Suslick K.S. Sonochemistry // Science. 1990. P.1439-1445.

21. Zhang, Z., Wang, G., Nie, Y., \& Ji, J. (2016). Hydrodynamic cavitation as an efficient method for the formation of sub-100 nm O/W emulsions with high stability. Chinese Journal of Chemical Engineering, 24(10), P. 1477-1480.

22. Agrawal, N., Maddikeri, G. L., \& Pandit, A. B. (2017). Sustained release formulations of citronella oil nanoemulsion using cavitational techniques. Ultrasonics Sonochemistry, 36, P. 367-374.

23. Carpenter, J., George, S., \& Saharan, V. K. (2017). Low pressure hydrodynamic cavitating device for producing highly stable oil in water emulsion: Effect of geometry and cavitation number. Chemical Engineering and Processing: Process Intensification, 116, P. 97-104.

24. Crudo D., Bosco V., Cavaglià G., Mantegna S., Battaglia L., Cravotto G. Process Intensification in the food industry: hydrodynamic and acoustic cavitation in fresh milk treatment. Process intensification // AgroFOOD Industry Hi Tech. 2014. Vol. 25(1). P.55-59.

25. Matsumiya K., Horiguchi S., Kosugi T., Mutoh T.A., Nambu Y., Nishimura K., MatsumuraY. Effects of heat treatment and homogenization on milk fat globules and proteins in whipping creams // Elsevier. Food Structure. 2017. Vol. 12. P. 94-102.

26. Aval M.Z., Akbarian M., Hosseinpou A. Nano cavitation: a novel technology in food science to improve edible oil, nanoemulsion, dairy production and water treatment: A REVIEW // Indian Journal of Fundamental and Applied Life Sciences ISSN: 22316345 (Online) An Open Access, Online International Journal Available at www.cibtech.org/sp.ed/jls/2016/02/jls.htm, 2016. Vol. 6 (S2), P. 651-656.

27. Phipps L. W. Cavitation and separated flow in a simple homogenizing valve and their influence on the break-up of fat globules in milk // Journal of Dairy Research. Published online: 01 June 2009.

28. Vitenko T., Drozdziel P., Horodyskyi N. Numerical modeling of a static cavitation module // Applied Mechanics and Materials. 2016. P. 64-69.

29. Vitenko T., Drozdziel P., Gorodysky N. Erosive Impact Of Vapor-Gas Phase On The Working Surface In Hydrodynamic Type Cavitation Mode // Diagnostyka. 2016. Vol.17. №.1. P.3-8. 
30. Jasinska M., Bałdyga J., Cooke M., Kowalski A.J. Specific features of power characteristics of in-line rotor-stator mixers // Chemical Engineering and Processing. 2015. Vol. 91. P.43-56.

31. Qin C., Chen C., Xiao Q., Yang N., Yuan C., Kunkelmann C., Cetinkaya M., Frank K. CFD-PBM simulation of droplets size distribution in rotor-stator mixing devices, 2016.38P. 